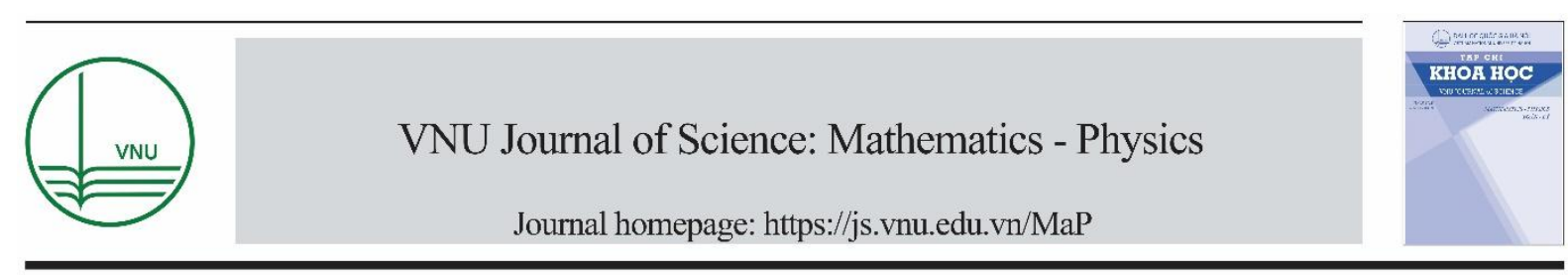

Original Article

\title{
Distribution of Sodium and Diffusion Mechanism in Sodium Silicate Liquid
}

\author{
Nguyen Thi Thanh $\mathrm{Ha}^{*}$ \\ Hanoi University of Science and Technology, 1 Dai Co Viet, Hanoi, Vietnam
}

Received 19 May 2019

Revised 09 June 2019; Accepted 15 June 2019

\begin{abstract}
In this study, molecular dynamic simulation is employed to study the structural properties and diffusion mechanism in sodium silicate $\left(\mathrm{Na}_{2} \mathrm{O} .4 \mathrm{SiO}_{4}\right)$. Structural characteristics are clarified through the pair radial distribution function, distribution of $\mathrm{SiO}_{\mathrm{x}}$ coordination units and network structure. The simulation results reveal that the structure of $\mathrm{Na}_{2} \mathrm{O} .4 \mathrm{SiO}_{4}$ liquid consists of one $\mathrm{Si}-\mathrm{O}$ network that is mainly formed by $\mathrm{SiO}_{4}$ units. The spatial distribution of sodium is non-uniform; sodium tends to be in the non-bridging oxygen-simplexes and in larger-radius simplex. Moreover, the sodium density for non-bridging oxygen region is significantly higher than the one for other regions. The study results also show that $\mathrm{Si}$ and $\mathrm{O}$ diffuse by bond break-reformation mechanism, while the motion of $\mathrm{Na}$ consists of two parallel processes. Firstly, $\mathrm{Na}$ atoms hop from one to another $\mathrm{O}$ within a disordered network where each bridging oxygen (BO) has one site, while a non-bridging oxygen (NBO) possesses two sites. The average resident time for Na staying near NBO is much longer than that near BO.
\end{abstract}

Keywords: Simulation, structure, mechanism diffusion, sodium-silicate.

\section{Introduction}

Glass-forming mixtures of $\mathrm{SiO}_{2}$ with an alkali oxide are the important materials group that has been widely applied in many fields such as: microelectronics, medicine (bio-material), high technology materials. Therefore, understanding their structure and dynamical properties is fundamentally necessary [1-3]. By using the neutron, X-ray diffraction (XRD) techniques, magic-angle spinning (MAS) nuclear magnetic resonance (NMR) $[4,5]$ and simulation methods $[6,7]$ observed the change in structure of alkali silicate. Namely, the addition of alkali oxides into pure silica $\left(\mathrm{SiO}_{2}\right)$ disrupts the basic silica network by breaking part of the Si-O bonds, creating non bridging oxygen (NBO). The percentage of NBOs in the system increases with the alkali oxide concentration $[8,9]$. In the multicomponent alkali

\footnotetext{
${ }^{*}$ Corresponding author.

Email address: ha.nguyenthithanh1@hust.edu.vn
}

https//doi.org/ 10.25073/2588-1124/vnumap.4354 
silicates, the "mixed alkali effect" refers to a drastic change of the relaxation dynamics. The ionic diffusivity of both alkali ions decreases in the mixed alkali composition; compared to the corresponding single-alkali silicate [10]. The effect is caused by mutual interception of jump paths of both kinds of mobile alkali ions. So the cooperative forward-correlated jumps are blocked and the immobilization of faster ions is responsible for the mixed alkali effect [11]. Moreover, alkali atom is found to migrate predominantly within NBO rich region in the system [8] and the diffusion of the alkali atoms mobility that is much higher than that of the silicon and oxygen atoms forming a tetrahedral network structure [12]. According to studies, the alkali atoms can easily move in preferential pathways, also called "channels". Thus, the fast transport of the alkali ions in silicate melt was explained $[13,14]$. The partial $\mathrm{Na}-\mathrm{Na}$ structure factor has a pre-peak at a wave vector $\mathrm{q}=0.95 \AA^{-1}$. These experimental results support a mechanism of ionic transport channel in alkali silicate $[15,16]$. The preferential pathways have been found via simulation method. The simulation box is divided into cubes and then they calculated the number density of the sodium atoms in each of these small boxes. The research results show that $50 \%$ of the cubes have been visited by sodium ions [17] and most of the Na motion occurs in $6 \%$ subsets of the total available space However, the boxes are fixed and placed in the space simulation box.

In this paper, we have calculated the statistical and dynamical distribution of sodium through the $\mathrm{Si}$ $\mathrm{O}$ network of $\mathrm{Na}_{2} \mathrm{O} .4 \mathrm{SiO}_{4}\left(\mathrm{NS}_{4}\right)$ liquid via simplex method. Furthermore, we focus on two diffusion mechanisms of network former and modifier atoms.

\section{Computational method}

MD simulation is carried out for $\mathrm{Na}_{2} \mathrm{O} .4 \mathrm{SiO}_{2}$ (NS4) melts at pressure of $0.1 \mathrm{MPa}$ and temperature of $1873 \mathrm{~K}$. The model contains 1066 Sodium, 2132 Silicon and 4797 Oxygen atoms. We apply the interatomic potential including two-body and three-body terms. This potential provided the reproduction of structure of silicate crystals and pressure dependence of transport properties of silicate liquid. More details about the applied potential can be found elsewhere $[18,19]$. The constructed models have been relaxed for long times until reach the equilibrium. Afterward, we perform additional runs of $150 \mathrm{ps}$ to produce a series of configurations separated by $10 \mathrm{ps}$. The dynamical and structural data are collected from these configurations.

The simplex is a sphere passing center of four oxygen atoms (NBO or BO) without $\mathrm{Si}$ or O atom inside. The simplex has no fixed radius. The radius of simplex depends only on position of four atoms. The simplexes consist of two main types: void-simplex (the sphere passing four atoms without any atom inside) and Na-simplex (the sphere passing four atoms and containing one or many sodium atoms). The Fig.1a, b presents void-simplex. The Fig.1c presents the Na-simplex. The characteristic of simplexes is determined by radius and type (BO, NBO) atom that sphere passing and the number of sodium inside.

a

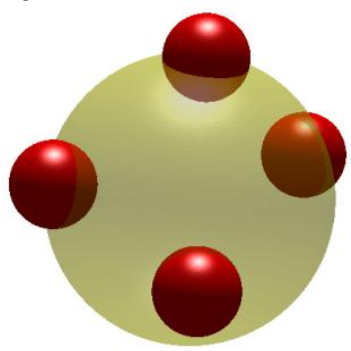

$\mathrm{b}$

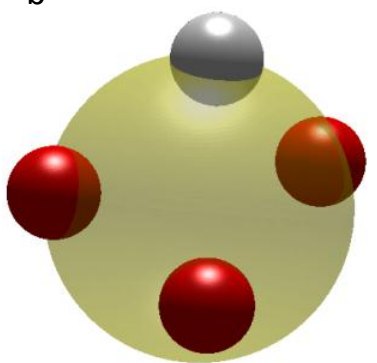

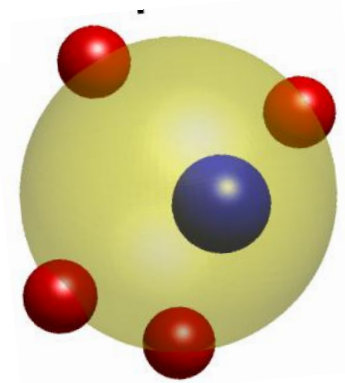

Fig 1. The schematic illustration of the simplex: void-simplex $(a, b)$; and Na-simplex $(c)$. Here the red, gray and blue spheres represent the NBO, BO and $\mathrm{Na}$ atoms, respectively; the circle represents the simplex sphere. 


\section{Results and discussion}

Table 1. the inter-atomic distances ( $r_{\mathrm{AB}} \AA$ ) calculated from PRDF of sodium silicate

\begin{tabular}{ccccccc}
\hline Reference & $r_{\mathrm{OO}}$ & $r_{\mathrm{SiO}}$ & $r_{\mathrm{ONa}}$ & $r_{\mathrm{SiSi}}$ & $r_{\mathrm{SiNa}}$ & $r_{\mathrm{NaNa}}$ \\
\hline $\mathrm{NS}_{4}$ & 2.62 & 1.62 & 2.15 & 3.12 & 3.20 & 3.32 \\
{$[20,21]$} & 2.62 & 1.62 & 2.29 & 3.05 & 3.5 & $2.6,3.05$ \\
{$[2]$} & 2.35 & 1.65 & & 3.12 & & \\
\hline
\end{tabular}

Firstly, we examine the structural and dynamical characteristics in $\mathrm{NS}_{4}$ liquid. Table 1 lists the interatomic distances calculated from PRDF and these results are compared with experimental data [2, 2021] for comparison. As seen, although $r_{S i N a}$ and $r_{N a N a}$ show some discrepancies, the constructed models are well consistent with experiments. In particular, the simulation reproduces the experimental data for $r_{O O}, r_{\mathrm{SiO}}, r_{\mathrm{SiSi}}$ and $r_{\mathrm{NaO}}$. The fraction of $\mathrm{SiO}_{\mathrm{x}}$ and different oxygen types is shown in Table 2 . The structure comprises $\mathrm{SiO}_{4}$ tetrahedrons and a small amount of $\mathrm{SiO}_{3}$. Close values of $D_{O}$ and $D_{S i}$ evidence the bond break-reformation mechanism for network atom [22]. Accordingly, the collective movement of network atoms occurs when a Si-O bond is broken and then a new bond is formed. This leads to rearrangement in the Si-O network and that Si moves cooperatively with its coordinated O. From Table 2 follows that fast $\mathrm{Na}$ move inside a network of slow atoms.

Table 2. Fraction of $\mathrm{SiO}_{\mathrm{x}}$ units and different oxygen types.

\begin{tabular}{cccccc}
\hline Units $\mathrm{SiO}_{\mathrm{x}}$ & Fraction of $\mathrm{SiO}_{\mathrm{x}}$ & Oxygen & $\begin{array}{c}\text { Fraction of type } \\
\text { Oxygen }\end{array}$ & $\begin{array}{c}\text { Diffusion coefficient, } \\
{\left[\mathrm{cm}^{2} / \mathrm{s}\right]}\end{array}$ \\
\hline $\mathrm{SiO}_{3}$ & 0.0041 & $\mathrm{FO}$ & 0.0007 & $\mathrm{D}_{\mathrm{O}}$ & $8.50 \times 10^{-8}$ \\
$\mathrm{SiO}_{4}$ & 0.9958 & $\mathrm{NBO}$ & 0.2225 & $\mathrm{D}_{\mathrm{Si}}$ & $6.50 \times 10^{-8}$ \\
$\mathrm{SiO}_{5}$ & 0.0001 & $\mathrm{BO}$ & 0.7767 & $\mathrm{D}_{\mathrm{Na}}$ & $4.74 \times 10^{-5}$ \\
\hline
\end{tabular}

To determine the temporal locations of $\mathrm{Na}$ we calculate the distances between every $\mathrm{Na}$ and network atoms. Then we determine a network atom that is separated from the given $\mathrm{Na}$ at a shortest distance. $\mathrm{We}$ assume that this $\mathrm{Na}$ is located in a site near the finding network atom. The calculation result is shown in Table 3. We observe that all $\mathrm{Na}$ are located in sites near $\mathrm{O}$ atoms and no one is present near $\mathrm{Si}$ atoms. This is due to both $\mathrm{Si}$ and $\mathrm{Na}$ are positively charged ions. For the convenience of discussion, we refer the phrase 'sites near $\mathrm{O}$ atoms' as 'near $\mathrm{O}$ atoms' for short. Although the number of $\mathrm{BO}$ more than three times excesses that of NBO, about $75 \%$ of $\mathrm{Na}$ is near NBO. This can be interpreted by the fact that $\mathrm{Na}$ is bonded to NBO much stronger than to BO because of NBO is connected only with one Si. From this follows that $t_{N B O}$ is much longer than $t_{B O}$, where $t_{B O}, t_{N B O}$ is the average resident time for Na being near $\mathrm{BO}$ and NBO, respectively. Furthermore, we observe that the number of $\mathrm{Na}$ located near an $\mathrm{O}$ varies from 0 to 1 when this $\mathrm{O}$ is $\mathrm{BO}$ and from 0 to 2 if it is NBO. There are some exceptions, for instance, three Na are present near NBO. However such cases occur very rarely. From this follows that each NBO has two sites, while a $\mathrm{BO}$ possesses one site. The total number of sites is calculated from the number of $\mathrm{NBO}$ and $\mathrm{BO}$. For the configuration at $t=0$ it equals to 5870 which is more than five times larger total number of $\mathrm{Na}$. This fact indicates that the simple vacancy mechanism is not appropriate for diffusion of sodium in $\mathrm{NS}_{4}$ liquid.

Using simplex method to identify the distribution of sodium in network $\mathrm{Si}-\mathrm{O}$, the results present Table 4. The most of $\mathrm{NBO}^{4}$-simplexes (four atoms are $\mathrm{NBO}$ ) and $\mathrm{BONBO}^{3}$ - simplexes (three $\mathrm{NBO}$ and one $\mathrm{BO}$ ) have the existence of $\mathrm{Na}$ atoms inside. The fraction of $\mathrm{Na}$ decreases with increasing number of BO. The result is consistent with the data in Table 3. 
Table 3. Spatial distribution of $\mathrm{Na}$ in Si-O network. $m_{N a}$ is the number of $\mathrm{Na}$ which are located near $\mathrm{NBO}$ or $\mathrm{BO}$; $m_{B O}, m_{N B O}$ is the number of $\mathrm{BO}$ and $\mathrm{NBO}$, respectively.

\begin{tabular}{ccccccc}
\hline \multirow{2}{*}{$\mathrm{m}_{\mathrm{Na}}$} & \multicolumn{2}{c}{$\mathrm{t}=0 \mathrm{ps}$} & \multicolumn{2}{c}{$\mathrm{t}=100 \mathrm{ps}$} & \multicolumn{2}{c}{$\mathrm{t}=140 \mathrm{ps}$} \\
\cline { 2 - 7 } & $\mathrm{m}_{\mathrm{BO}}$ & $\mathrm{m}_{\mathrm{NBO}}$ & $\mathrm{m}_{\mathrm{BO}}$ & $\mathrm{m}_{\mathrm{NBO}}$ & $\mathrm{m}_{\mathrm{BO}}$ & $\mathrm{m}_{\mathrm{NBO}}$ \\
\hline 0 & 3485 & 373 & 3468 & 394 & 3477 & 391 \\
1 & 243 & 570 & 252 & 555 & 243 & 549 \\
2 & 0 & 125 & 1 & 124 & 1 & 133 \\
3 & 0 & 1 & 0 & 3 & 0 & 2 \\
\hline
\end{tabular}

Table 4. Characteristic of simplex in sodium silicate.

\begin{tabular}{ccccc}
\hline Type of simplex & $\mathrm{m}_{\text {simplexes }}$ & $\begin{array}{c}\text { Fraction of } \\
\text { simplexes }\end{array}$ & $\begin{array}{c}\text { Simplexes with } \\
\mathrm{Na}\end{array}$ & $\begin{array}{c}\text { Fraction of } \\
\text { simplexes with Na }\end{array}$ \\
\hline $\mathrm{NBO}^{4}$ & 338 & 0.0179 & 327 & 0.9675 \\
$\mathrm{BONBO}^{3}$ & 1454 & 0.0770 & 1339 & 0.9209 \\
$\mathrm{BO}^{2} \mathrm{NBO}^{2}$ & 4011 & 0.2124 & 3396 & 0.8467 \\
$\mathrm{BO}^{3} \mathrm{NBO}$ & 6940 & 0.3675 & 4768 & 0.6870 \\
\hline
\end{tabular}

Moreover, the characteristic of simplex is determined by radius of simplex and the radius distribution of void simplex, 1Na-simplex and 2Na-simplex is illustrated in Fig 2. It reveals that the radius distributions have the Gaussian form and the position of the peak systematically shifts to right (larger radius) as a number of sodium inside simplex increases. So, we conclude that $\mathrm{Na}$ atoms tend to be in the NBO-simplex and simplex has larger radius. The motion of Na relates strongly to simplextype (BO or NBO).

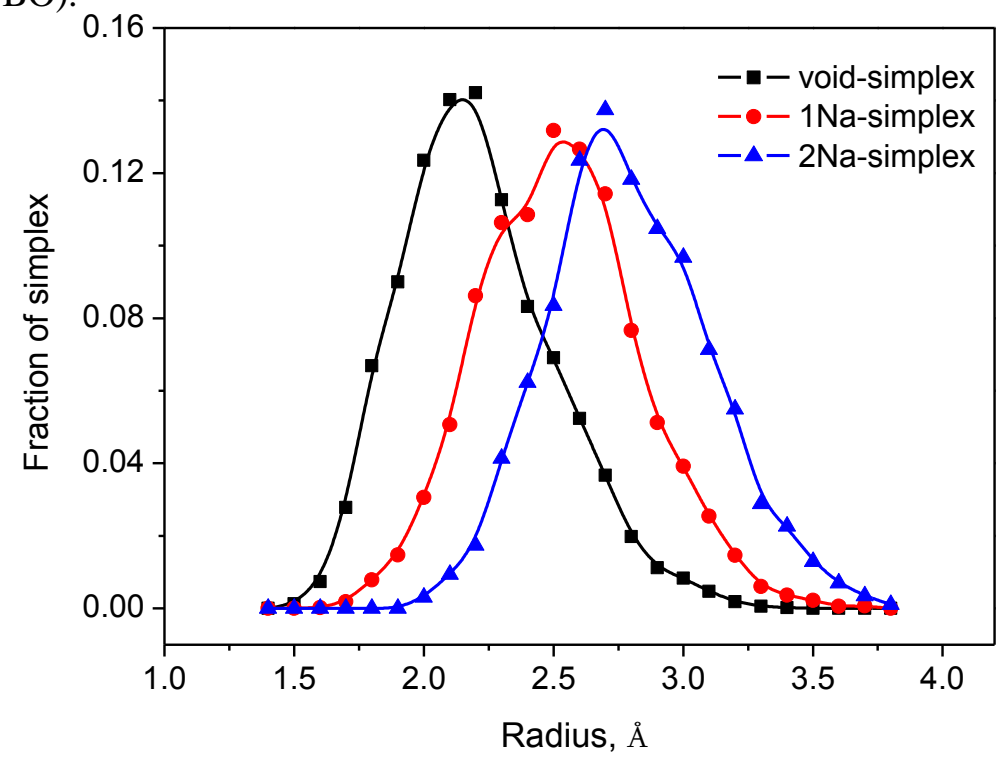

Fig 2. The radius distribution of void simplex, $1 \mathrm{Na}$-simplex and $2 \mathrm{Na}$-simplex. 
We also calculate the time dependence of fraction $\mathrm{X}^{\mathrm{n}} / \mathrm{X}$, where $\mathrm{X}$ is the total number of $\mathrm{BO}$ (or $\mathrm{NBO}$ ); $\mathrm{X}^{\mathrm{n}}$ is the number of $\mathrm{BO}$ (or $\mathrm{NBO}$ ) near which $n$ sodium atoms are present. The result is presented in Fig.3. As expected, $\mathrm{BO}^{1} / \mathrm{BO}$ is significantly smaller than $\mathrm{NBO}^{1} / \mathrm{NBO}$ and $\mathrm{NBO}^{2} / \mathrm{NBO}$. Very small fraction $\mathrm{BO}^{1} / \mathrm{BO}$ confirms the fact that $\mathrm{Na}$ atoms stay near $\mathrm{NBO}$ in average for much longer time than that near BO. Thus we can conclude that $\mathrm{Na}$ atoms move from sites to sites located near $\mathrm{O}$ atoms and the resident time for Na near NBO is much longer than that near BO.

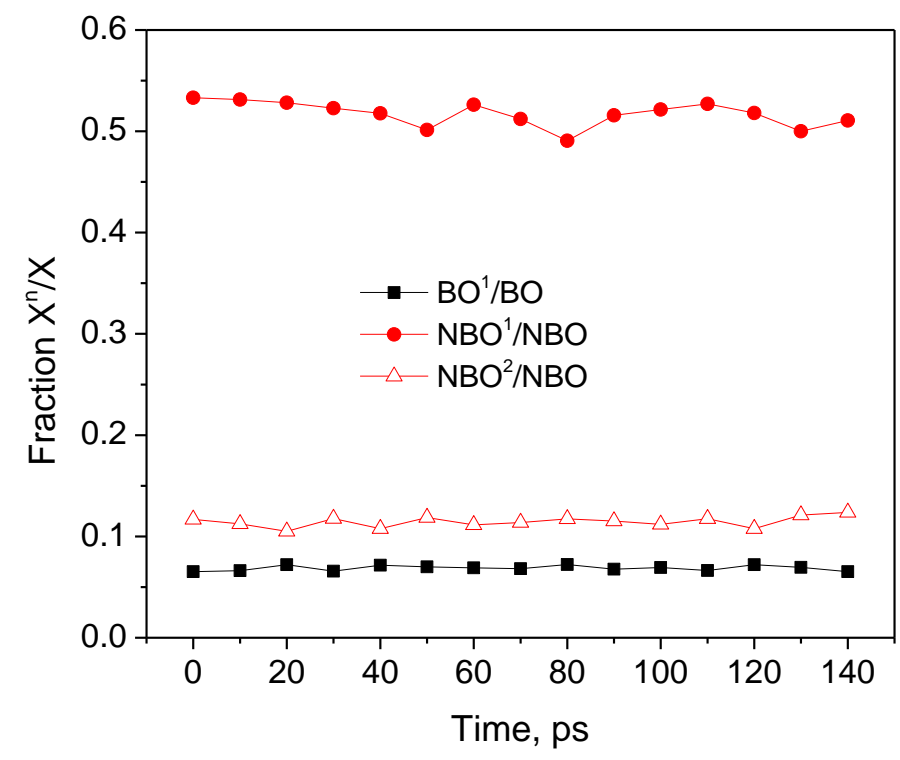

Fig.3. Fractions $\mathrm{X}^{\mathrm{n}} / \mathrm{X}$ as a function of time.

Next, we examine the status (BO or NBO) and occupancy for O. The occupancy for $\mathrm{O}$ is busy if $\mathrm{Na}$ is present near this $\mathrm{O}$, otherwise it is free. We consider 15 configurations separated by $10 \mathrm{ps}$. For every $\mathrm{O}$ we count how many times when its status is $\mathrm{BO}\left(M_{B O}\right)$ and the occupancy for $\mathrm{O}$ is busy $\left(M_{N a}\right)$. Obviously, $M_{N a}$ and $M_{B O}$ vary from 0 to 15 . The plots of number of $\mathrm{O}$ versus $M_{N a}$ and $M_{B O}$ are shown in Fig 4. We observe that about $14 \%$ of $\mathrm{O}$ atoms have $M_{B O}$ from 1 to 14 . These $\mathrm{O}$ undergo transformations from NBO to $\mathrm{BO}$ and vice versa during the time $t_{o b s}$. When the transformations of $\mathrm{O}$ status occur, $\mathrm{Na}$ atoms will be redistributed between different $\mathrm{O}$ atoms. In particular, as $\mathrm{NBO}$ transforms to $\mathrm{BO}$, the $\mathrm{Na}$ atoms located near this NBO move to other $\mathrm{O}$ atoms. In the case when $\mathrm{BO}$ transforms to NBO, many $\mathrm{Na}$ atoms replace to the transformed $\mathrm{BO}$. Thus, the motions of $\mathrm{Na}$ is strongly correlated with the one of network atom. We also mention that $86 \%$ of $\mathrm{O}$ atoms have $M_{B O}$ equal to 0 or 15 . This means that during the time $t_{o b s}$ the redistribution of $\mathrm{Na}$ happens with a small number of $\mathrm{O}$ atoms.

As shown in Fig.4, most of $\mathrm{O}$ atoms possess $M_{\mathrm{Na}}$ varied from 1 to 12 indicating a high rate of $\mathrm{Na}$ jumping. However there are $27 \%$ of $\mathrm{O}$ atoms having $M_{N a}=0$, i.e. Na atoms do not enter sites near these $\mathrm{O}$. This indicates the non-uniform spatial distribution of $\mathrm{Na}$. Note that two $\mathrm{O}$ atoms connected to a common Si also form a linkage with $r_{l k}=3.6 \AA$. Further, we observe that Na located near a beforehoping $\mathrm{O}$ can jump to one among neighbors of the before-hoping O. From this follows that Na atoms hop from $\mathrm{O}$ atom to its neighbors within the $\mathrm{O}$ network. The hoping may be blockaded when neighboring atoms are completely occupied by other $\mathrm{Na}$ atoms. 

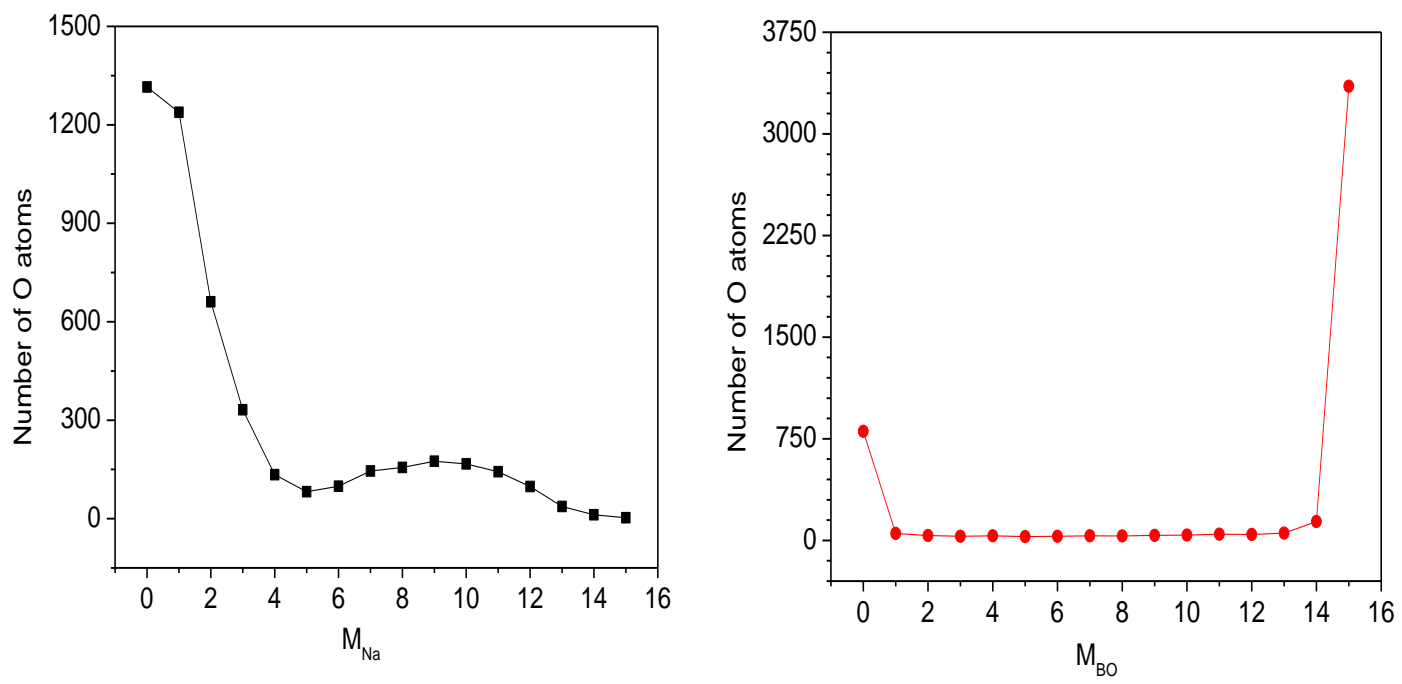

Fig.4. Plots of number of $\mathrm{O}$ versus $M_{N a}$ and $M_{B O}$. Here $M_{B O}, M_{N a}$ respectively is the number of times when the status is BO and the occupancy is busy.

\section{Conclusion}

The $\mathrm{NS}_{4}$ liquid is systematically analyzed on dynamics and structure by MD simulation. The results show that the structure of liquid consists of a network of $\mathrm{SiO}_{4}$ connected with each other via $\mathrm{BO}$. There is also a large amount of NBO, up to $22 \%$ of total Oxygen atoms. Furthermore, the distribution of sodium is determined via the simplex and the average resident time for Na staying near NBO or BO. It reveals that the density of Na for NBO- region is significantly larger than that for other region and sodium atoms can easily move in NBO regions. Two diffusion mechanisms of network former and modifier are discussed. : the network atoms diffuse by the bond break-reformation, while diffusion of $\mathrm{Na}$ consists of two parallel processes. First, $\mathrm{Na}$ atoms move from one to another $\mathrm{O}$ atom within a disordered $\mathrm{O}$ network where the majority of atoms have from 5 to 8 neighbors. The jump of $\mathrm{Na}$ is realized between two $\mathrm{O}$ atoms forming a linkage with $r_{l k}=3.6 \AA$. Second, Na atoms are redistributed between $\mathrm{O}$ atoms as the transformation of $\mathrm{O}$ status happens. Furthermore, we found that the $\mathrm{O}$ network comprises a number of sites where each BO has one site, while NBO possesses two sites.

\section{Acknowledgements}

The authors are grateful for support by the NAFOSTED Vietnam (Grant 103.01-2018.13).

\section{References}

[1] Gergely Molnár, Patrick Ganster, Anne Tanguy, Effect of composition and pressure on the shear strength of sodium silicate glasses: An atomic scale simulation study, Physical Review E 95 (2017) 043001.

[2] A.O. Davidenkoa, V.E. Sokol'skiia, A.S. Roika, I.A. Goncharovb, Structural Study of Sodium Silicate Glasses and Melts, Inorganic Materials 50 (12) ( 2014)1289.

[3] K. Konstantinou, D.M . Duffy, A.L. Shluger, Structure and luminescence of intrinsic localized states in sodium silicate glasses, Physical review B 94 (2016) 174202. 
[4] S.K. Lee, G.D. Cody, Y. Fei, B.O. Mysen, The effect of Na/Si on the structure of sodium silicate and aluminosilicate glasses quenched from melts at high pressure: A multi-nuclear (Al-27, Na-23, O-17) 1D and 2D solid-state NMR study, Chem. Geol. 229 (2006) 162-172.

[5] J.R. Allwardt, J.F. Stebbins, B.C. Schmidt, D.J. Frost, A.C. Withers, M.M. Hirschmann, Aluminum coordination and the densification of high-pressure aluminosilicate glasses, Am. Mineral. 90 (2005)1218-1222.

[6] E. Sunyer, P. Jund, R. Jullien, Matrix-controlled channel diffusion of sodium in amorphous silica,J. Phys.: Condens. Matter 15, L431-L437 (2003).

[7] M. Bauchy, M. Micoulaut, From pockets to c hannels: Density-controlled d iffusion in sodium silicates,Physical review B 83 (2011)184118.

[8] H. Jabraoui, Y. Vaills, A.Hasnaoui, M. Badawi, S. Ouaskit, Effect of Sodium Oxide Modifier on Structural and Elastic Properties of Silicate Glass, J. Phys. Chem. B 120 ( 2016) 13193.

[9] T.K. Bechgaard el al.., Structure and mechanical properties of compressed sodium aluminosilicate glasses: Role of non-bridging oxygens, Journal of Non-Crystalline Solids 441 (2016) 49-57.

[10] S.I. Sviridov, Diffusion of cations in sodium-potassium and sodium-barium silicate melts, Glass Physics and Chemistry 39 (2) (2013) 130-135.

[11] J. Habasaki, K.L. Ngai, Y. Hiwatari, "Cooperativity blockage" in the mixed alkali effect as revealed by moleculardynamics simulations of alkali metasilicate glass, Journal of chemical physics 121 (2) (2004) 925.

[12] G.N. Greaves, S. Sen, Inorganic glasses, glass-forming liquids and amorphizing solids, Adv. Phys. 56 (1) (2007).

[13] T. Voigtmann, J. Horbach, Slow dynamics in ion-conducting so dium silicate melts:Simulation and mo de-coupling theory, Europhys. Lett., 743 (2006) 459-465.

[14] Alfonso Pedone et al., A New Self-Consistent Empirical Interatomic Potential Model for Oxides, Silicates, and Silica-Based Glasses J. Phys. Chem. C. 112 (2008) 11034-11041.

[15] E. Sunyer, P. Jund, R. Jullien, Numerical investigation of ionic transport in glasses:the example of sodium in amorphous silica, J. Phys. Condens. Matter 15 (2003) S1659.

[16] P. Jund, W. Kob, R. Jullien, Channel diffusion of sodium in a silicate glass, Phys. Rev. B 64 (2001) 134303.

[17] A. Meyer, F. Kargk, J. Horbach, Channel diffusion in sodium silicate melts, Journal of Netron new 23 (3) (2012).

[18] H. Sakuma, K. Kawamura, Structure and dynamics of water on muscovite mica surfaces, Geochim. Cosmochim. Acta 73 (2009) 4100.

[19] F. Noritake, K. Kawamura, T. Yoshino, E. Takahashi, Molecular dynamics simulation and electrical conductivity measurement of $\mathrm{Na}_{2} \mathrm{O} .3 \mathrm{SiO}_{2}$ melt under high pressure; relationship between its structure and properties, J. NonCryst. Sol. 358 (2012) 3109

[20] M. Fabian, P. Jovari, E. Svab, Gy Meszaros, T. Proffen, E.Veress, Network structure of 0.7SiO2-0.3Na2O glass from neutron and x-ray diffraction and RMC modelling, J. Phys.: Cond. Matt., 19 (2007)

[21] N. Zotov, H. Keppler, The structure of sodium tetra-silicate glass from neutron diffraction, reverse Monte Carlo simulations and Raman spectroscopy, Phys. Chem. Minerals, 25 (1998) 259-267.

[22] E. Sunyer, P. Jund, R. Jullien, Characterization of channel diffusion in a sodium tetrasilicate glass via molecular-dynamics simulations, Phys. Rev. B 65 (2002) 214203 